Simone Lorena Quiterio 1

Célia Regina Sousa da Silva 1

Graciela Arbilla 1

Maria de Fátima Ramos Moreira 2

Ulisses César Araújo 2

Rita de Cássia O. da C. Mattos 2

Luiz Sérgio Cardoso Santos 2

\section{Controle das emissões de chumbo particulado no entorno de uma reformadora de baterias da cidade do Rio de Janeiro usando ar como indicador}

\author{
Control of lead emissions from a battery \\ repair shop in the city of Rio de Janeiro \\ by monitoring air quality
}

1 Departamento de Físico-Química, Instituto de Química, Universidade Federal do Rio de Janeiro. Av. Brigadeiro Trompovski s/n, Centro de Tecnologia, bloco A, Rio de Janeiro, RJ 21949-900, Brasil.

2 Centro de Estudos da Saúde do Trabalhador e Ecologia Humana, Escola Nacional de Saúde Pública, Fundação Oswaldo Cruz. Av. Leopoldo Bulhões 1480, Rio de Janeiro, $R J$ 21040-210, Brasil.

\begin{abstract}
Lead levels in the air surrounding a battery repair shop (BRS) located in the Olaria neighborhood (Rio de Janeiro, Brazil) were monitored from April to July 1999. Most of the sam-

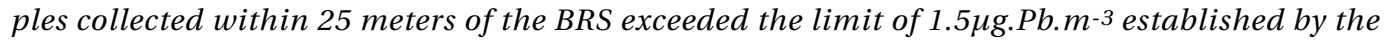
United States Environmental Protection Agency (EPA). These results were published in a previous article (Quiterio et al., 2001). In the current study, installation of a Venture ventilation system and some changes in the operational procedure were proposed. After the modifications in the BRS, a new monitoring campaign was performed (August and September, 2000). Three points were selected for air collection, corresponding to the most critical points found in the previous assessments. The new concentrations ranged from 0.8 to $17.6 \mu \mathrm{g} . \mathrm{Pb} . \mathrm{m}^{-3}$, showing that lead emissions into the environment had decreased adequately. However, concentration at the chimney exhaust was still higher than EPA limits, and further improvements in the installations and procedures are definitely needed.
\end{abstract}

Key words Lead; Environmental Monitoring; Environmental Exposure

Resumo No período entre abril a julho de 1999, foram realizadas determinações dos níveis de chumbo no ar ambiente nas proximidades de uma reformadora de baterias (RB) situada em Olaria (Rio de Janeiro, Brasil). A maioria das amostras coletadas a uma distância de até $25 \mathrm{~m}$ da

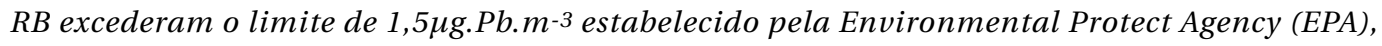
Estados Unidos. Esses resultados já foram publicados previamente (Quiterio et al., 2001). Neste trabalho, foram propostas a instalação de um sistema de exaustão Venture e algumas mudanças nos procedimentos operacionais. Após as modificações na $\mathrm{RB}$, foi realizada uma nova campanha de monitoramento (agosto e setembro de 2000). As coletas foram realizadas nos três pontos considerados mais críticos, segundo as determinações anteriores. As concentrações medidas estiveram no intervalo 0,8-17,6 $\mathrm{g}$.Pb.m-3, mostrando que as emissões de chumbo na atmosfera tiveram uma diminuição adequada. Contudo, a concentração na saída da chaminé é ainda maior que os limites estabelecidos pela EPA e, certamente, serão necessárias outras melhorias nas instalações e procedimentos.

Palavras-chave Chumbo; Monitoramento Ambiental; Exposição Ambiental 


\section{Introdução}

O chumbo tem alta toxicidade e uma tendência excepcional de se acumular no organismo humano (Baird, 1995). A presença natural do chumbo no meio ambiente não causa problemas à saúde, por se dar em pequenas concentrações (RTI, 1999). Entretanto, devido à ampla utilização nos processos industriais e conseqüente liberação para o ambiente, os níveis de chumbo no ar têm aumentado, tornando necessário o seu monitoramento.

Essas atividades industriais são freqüentemente realizadas em locais próximos a residências, aumentando os níveis ambientais de chumbo e expondo a população residente na circunvizinhança da fonte de emissão estacionária (FEE) a efeitos nocivos no organismo, especialmente no caso de crianças que são mais sensíveis à contaminação.

Recentes estudos em diversos países têm associado à exposição ambiental ao chumbo com efeitos adversos em diferentes sistemas do organismo humano, incluindo alterações nos sistemas neurológico, hematológico, metabólico e cardiovascular (Galvão \& Corey, 1989).

Nos países em desenvolvimento, as reformadoras de baterias (RB) são uma das principais fontes de emissão estacionária de chumbo, contaminando o ambiente, as populações e suas residências. Pequenas reformadoras de baterias, que têm se desenvolvido principalmente em países do terceiro mundo, representam um alto risco de contaminação para moradores de residências próximas, pois nelas são utilizados processos e tecnologia obsoleta; geralmente, as instalações são precárias e são ampliadas sem planejamento ou cuidados com a purificação do ar (Skerfving, 1993). Situações desse tipo ocorrem, por exemplo, em Koplan (Barbados), Nigéria, Trinidad e Tobago, Kingston (Jamaica), nos Andes (Equador) e em Taiwan, locais em que foram encontrados altos níveis de chumbo no sangue dos moradores de residências adjacentes (Matte et al., 1989; Yeh et al., 1996).

A emissão de chumbo acontece sobretudo nas atividades de empilhamento e encaixe das placas e solda dos terminais de chumbo. A manipulação das placas resulta no desprendimento de grandes quantidades de poeira (partículas) constituídas, particularmente, pelo chumbo sob a forma metálica e na forma de óxidos; reservando-se a emissão de fumos contendo o metal à soldagem (Quiterio, 2000).

Numa publicação recente (Quiterio et al., 2001) foram reportados valores de concentração de chumbo, no ar das vizinhanças de uma
RB situada no Rio de Janeiro, superiores aos limites estabelecidos pela Environmental Protection Agency (EPA) (RTI, 1999). O reconhecimento da necessidade e do compromisso com a melhoria da condição de vida dos trabalhadores e da população exposta ao chumbo emitido por essa reformadora de baterias impulsionou à continuidade deste trabalho.

Este novo estudo teve como objetivo avaliar, por meio do monitoramento ambiental, empregando-se como indicador o ar atmosférico, o nível de chumbo presente em regiões circunvizinhas a uma FEE, depois da adoção pela empresa de medidas de controle da poluição.

\section{Material e métodos}

\section{Localização e climatologia}

Segundo dados do IBGE (Fundação Instituto Brasileiro de Geografia e Estatística), a região estudada, localizada na zona norte da cidade do Rio de Janeiro, no bairro de Olaria, é cercada por planícies ao norte, possui uma população de aproximadamente dez mil habitantes, quatro escolas municipais e três hospitais no raio de até $500 \mathrm{~m}$ da reformadora (CICT, 1999). Nessa região, conforme a classificação de Köpper, o clima é Cwa (clima meso-térmico com chuvas de verão e verão quente), ocorrendo, diariamente, uma fina camada de poeira no ar (Marques, 1972). Consoante as informações climáticas da estação meteorológica localizada no Flamengo (Zona Sul), na cidade do Rio de Janeiro, a média da temperatura, do índice pluviométrico e da umidade no ano de 1999 foram $23,7 \circ \mathrm{C}, 1172,9 \mathrm{~mm}^{3}$ de chuva e $79 \%$, respectivamente, enquanto no ano de 2000 foram $24,2^{\circ} \mathrm{C}$, $652 \mathrm{~mm}^{3}$ e $75 \%$, respectivamente. Nos ventos, predominou a direção nordeste e, nos dias de chuva, a direção sudoeste. Nos dias de chuva, o particulado é removido por deposição úmida, não ficando suspenso no ar (Pinhel, 2000).

\section{Instrumental}

O sistema de coleta do ar atmosférico foi constituído por uma bomba de médio volume com vazão de 20L.min-1, marca Sibata, modelo IP20T, suporte para filtro, filtro de nitrocelulose Millipore (Bedford, Massachusetts, Estados Unidos) com $55 \mathrm{~mm}$ de diâmetro e poro de 0,8 $\mathrm{mm}$, e ainda tripé e separador de partículas.

As análises foram feitas num espectrômetro de absorção atômica Zeeman 5100, equipado com um forno de grafite HGA-600 e um amostrador automático As-60, todos Perkin-El- 
mer (Norwalk, Connecticut, Estados Unidos). Foram utilizados tubos revestidos com grafite pirolítico (Perkin-Elmer Part No. B010-9322) com plataforma de grafite pirolítico (Perkin-Elmer Part No. B010-9324). A uma lâmpada de cátodo oco de chumbo (Perkin-Elmer Part No. N066-1299) foi aplicada uma corrente de 10mA; o comprimento de onda e a largura da fenda foram ajustados para $283,3 \mathrm{~nm}$ e $0,7 \mathrm{~nm}$, respectivamente. A avaliação do sinal corrigido Zeeman foi realizada por medidas de absorvância integrada, área de pico (AP).

\section{Materiais e reagentes}

Todo o material plástico e vidraria utilizada ficaram imersos por um período mínimo de 24 horas em solução de Extran (Merck - Elmsford, Nova Iorque, Estados Unidos) a 5\%(v/v), enxaguados abundantemente em água corrente e novamente imersos por, pelo menos, 48 horas em solução de ácido nítrico (Merck) 10\% (v/v), para descontaminação. Em seguida, foram enxaguados copiosamente com água ultrapura do tipo Milli-Q (Millipore) e secos à 40॰C (Moreira, 1993).

Todos os reagentes utilizados foram, pelo menos, de grau analítico (PA). O padrão foi preparado diariamente por diluições apropriadas da solução estoque de $1.000 \mu \mathrm{g}$. $\mathrm{mL}^{-1}$ de chumbo da ampola Titrisol - Merck em ácido nítrico $0,2 \%(\mathrm{v} / \mathrm{v})$, usando-se água previamente purificada em sistema Milli-Q (Millipore).

\section{Metodologia de amostragem}

A escolha dos pontos de coleta obedeceu às normas estabelecidas pela EPA, que se aplicam na amostragem do ar em ambientes externos, quando a fonte de emissão é estacionária. Desta forma, há algumas recomendações que devem ser observadas: altura ideal, topografia, meteorologia, dispersão e deposição do chumbo (EPA, 1998).

A amostragem foi realizada nas seguintes distâncias em relação ao centro da RB: sudeste $-6 \mathrm{~m}$; sudeste $-17 \mathrm{~m}$; nordeste $-13 \mathrm{~m}$; nordeste $-17,5 \mathrm{~m}$; sudoeste $-16 \mathrm{~m}$; noroeste $-24 \mathrm{~m}$; sudoeste $-500 \mathrm{~m}$; nordeste $-500 \mathrm{~m}$; noroeste $500 \mathrm{~m}$ e sudeste $-500 \mathrm{~m}$, conforme pode ser verificado na Figura 1.

Antes da instalação do sistema de exaustão, foram realizadas coletas de ar em seis dias diferentes, no período de abril a julho de 1999. Para cada dia, foram realizadas três amostragens nos seis pontos situados até $25 \mathrm{~m}$ de distância da RB e uma coleta em quatro pontos distintos a 500m da RB (Quiterio et al., 2001).

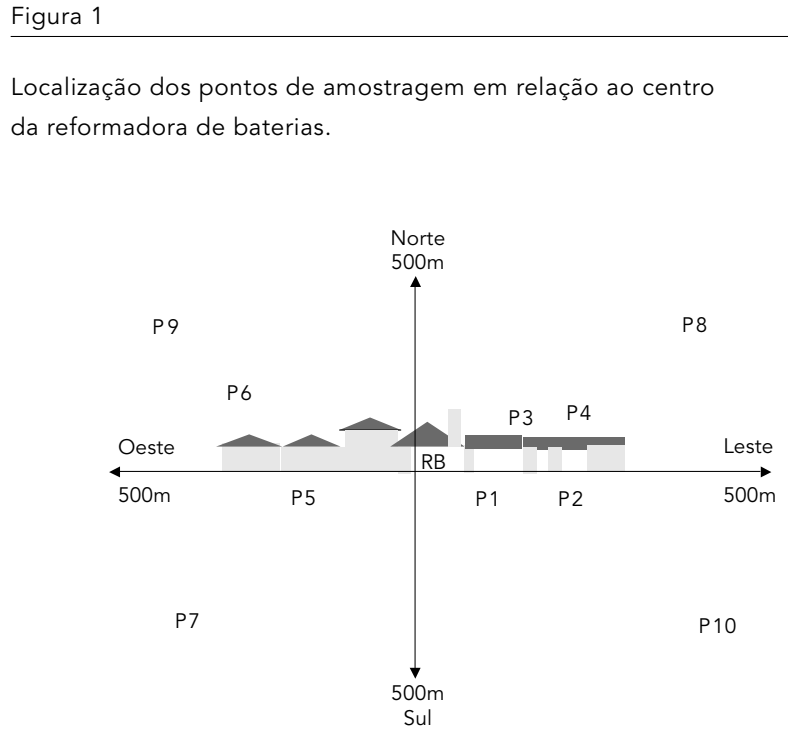

$\mathrm{P} 1=6 \mathrm{~m}$ - sudeste; $\mathrm{P} 2=17 \mathrm{~m}$ - sudeste; $\mathrm{P} 3=13 \mathrm{~m}$ - nordeste; $\mathrm{P} 4=17,5 \mathrm{~m}$ nordeste; $P 5=16 \mathrm{~m}$ - sudoeste; $\mathrm{P} 6=24 \mathrm{~m}$ - nordeste; $\mathrm{P} 7=500 \mathrm{~m}$ - sudoeste; $P 8=500 m-$ nordeste; $P 9=500 m-$ noroeste $P 10=500 m-$ sudeste.

Após as modificações na $\mathrm{RB}$, foram realizadas duas coletas em três pontos distintos situados num raio até $25 \mathrm{~m}$ (pontos 3 , 4 e 6 ) nos meses de agosto e setembro de 2000.

O tempo de coleta foi de cinco horas. Após este período, o amostrador foi desconectado e o papel de filtro cuidadosamente retirado do porta-filtros e colocado em frascos de polietileno para posterior análise.

Um local próximo à praia que não apresenta indústrias, fábricas, ou qualquer outra fonte de emissão estacionária de chumbo, no Recreio dos Bandeirantes, Rio de Janeiro, foi usado como área de controle para este trabalho.

\section{Análise quantitativa}

Foi utilizada a técnica de espectrometria de absorção atômica no forno de grafite (EAA-FG) que, em razão de sua alta sensibilidade, é amplamente aplicada na análise de material particulado em papéis de filtro, para a determinação quantitativa de metais (Vahter \& Slorach, 1990).

Os filtros foram colocados, cuidadosamente, em frascos de polietileno, dissolvidos com $3 \mathrm{ml}$ de ácido nítrico concentrado, em temperatura ambiente por duas horas, e levados a 50ml com água Milli-Q. A amostra foi analisada por EAA-FG, segundo o Método 3015A (EPA, 1999).

A acurácia do método foi verificada por intermédio da análise de filtros de referência cer- 
tificados (SRM 3087a, National Institute of Standards Technology, Estados Unidos, valor certificado: 40,38 \pm 0,92 $\mu$ g.filtro-1) (NIST, 1997). A concentração de chumbo encontrada no material certificado foi 37,98 $\pm 6,43 \mu$ g. filtro-1 (Quiterio, 2000). Entre o valor certificado e o experimental não foi encontrada diferença estatisticamente significativa (Student's $t$ test, 95\%).

O limite de detecção do método foi estimado em $0,03 \mu \mathrm{g} \cdot \mathrm{m}^{-3}$, fazendo dez determinações em um filtro não amostrado e diluído (Quiterio, 2000).

A curva de calibração foi construída seguindo-se padrões $\left(0-50 \mu\right.$ g chumbo $\left.L^{-1}\right)$ preparados com base em uma solução estoque (Tritisol - Merck). Os coeficientes de correlação obtidos em todas as curvas foram melhores que 0,9985 (Quiterio, 2000).

\section{Resultados e discussão}

\section{Concentração de chumbo} nas amostras de ar (Pb-Ar)

A dispersão, deposição e distribuição do chumbo no ambiente dependem, fundamentalmente, das condições meteorológicas e dos parâmetros de emissão da fonte, como velocidade e temperatura de emissão do poluente (EPA, 1998).

A difusão do chumbo está relacionada de maneira ampla com as mudanças de correntes eólicas e de temperatura existentes na camada de ar. O vento, um importante parâmetro meteorológico, é resultante das diferenças de pressão em virtude do aquecimento ou resfriamento da atmosfera pelo sol, e é capaz de espalhar o chumbo numa determinada região e transportá-lo para longe de seu ponto de emissão (Landrigan et al., 1975).

Verificou-se, nas avaliações anteriores, realizadas em dez pontos de coleta, que o chumbo se dispersa no raio de $25 \mathrm{~m}$ da $\mathrm{RB}$, na faixa de concentração de 0,07 a $183,3 \mu \mathrm{gPb} \cdot \mathrm{m}^{-3}$. As maiores concentrações de chumbo foram encontradas a treze metros na direção nordeste

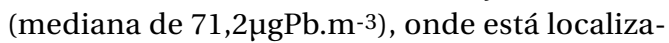
da a principal saída de ar do setor de produção, sugerindo que as concentrações encontradas estão relacionadas com as atividades desenvolvidas na RB e à direção predominante do vento (nordeste) (Quiterio et al., 2001).

Nessa primeira avaliação do ar externo, três pontos de coleta de ar foram considerados os mais críticos, pois excederam o limite tolerado

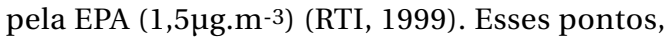
identificados como 3, 4 e 6 (saída da chaminé ar tratado pelo SE; varanda da residência - fun- dos da RB e galpão de depósito - ao lado esquerdo, respectivamente), foram avaliados uma vez mais após as modificações na RB.

No raio de $500 \mathrm{~m}$, as concentrações foram equivalentes àquela do branco (Quiterio et al., 2001).

\section{Concentração de chumbo após \\ instalação do sistema de exaustão}

Foram propostas modificações no processo de produção e melhorias das instalações, a fim de que houvesse uma redução na emissão do metal.

No processo de produção foi implementado o uso de equipamento de proteção individual e a lavagem do piso, visto que o transporte de placas leva inevitavelmente à dispersão de pó no piso da fábrica.

As instalações foram melhoradas com a colocação de um SE Venture, bastante eficiente no que se refere à captação de pó ou de gases poluentes. O princípio de funcionamento desse equipamento é baseado na impactação da água com os gases. $\mathrm{O}$ ar (contaminado) atravessa, de baixo para cima, uma camada de enchimento, que em certos casos lembra uma colmeia. Tal camada recebe o borrifo de água de aspersores (bicos) colocados acima dela. Esta camada de enchimento assegura a maior área de contato possível dos gases com a água despejada pelos bicos pulverizadores, funcionando, portanto, em contra-corrente. Pela impactação dos gases com a água, as partículas que se chocam se depositam por difusão, aumentam de tamanho e a sua coleta torna-se mais fácil. Logo, os quatro mecanismos de coleta são a impactação, a interceptação, a difusão e a condensação.

Após a instalação do SE, monitorou-se o ar tratado emitido para o ambiente para se avaliar a eficiência das modificações propostas. Como citado anteriormente, três pontos de coleta de ar, os pontos mais críticos do ar externo encontrados nas avaliações anteriores, foram monitorados: Ponto 3 (saída da chaminé - ar tratado pelo SE), Ponto 4 (varanda da residência - fundos da RB) e Ponto 6 (galpão de depósito - ao lado esquerdo), a saber, nordeste $13 \mathrm{~m}$; nordeste $-17,5 \mathrm{~m}$; noroeste $-24 \mathrm{~m}$, respectivamente.

Os resultados da primeira avaliação (sem SE - período de abril a julho de 1999) e da segunda e terceira avaliações (agosto e setembro de 2000) são apresentados e comparados na Figura 2. Estes valores correspondem à mediana de três monitoramentos.

$\mathrm{O}$ valor encontrado para a amostra da área controle (Recreio dos Bandeirantes, Rio de Ja- 
neiro), considerada como branco, foi de 0,07 $\mu \mathrm{gPb} . \mathrm{m}^{-3}$, que é inferior ao limite tolerado pela EPA de 1,5 $\mu \mathrm{gPb} . \mathrm{m}^{-3}$ (RTI, 1999).

No Ponto 4, foram obtidos os valores 1,2 e $0,9 \mu \mathrm{gPb} . \mathrm{m}^{-3}$, para os meses de agosto e setembro, respectivamente. No Ponto 6, os valores de 0,8 e $1,1 \mu \mathrm{gPb} . \mathrm{m}^{-3}$, para os mesmos meses. Esses resultados demonstram que, após a instalação do SE, houve importante redução dos níveis de chumbo emitidos para o ambiente. Nos pontos 4 e 6 a redução foi entre 42 e $57 \%$, o que possibilitou obter níveis médios de $\mathrm{Pb}$ menores que o valor de referência de $1,5 \mu \mathrm{gPb} . \mathrm{m}^{-3}$ (RTI, 1999). Entretanto, os valores obtidos (medianas) são ligeiramente superiores aos valores médios informados na literatura (médias no

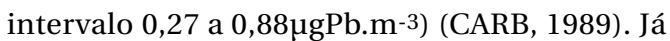
no ponto 3 , foram obtidos os valores $12,1 \mathrm{e}$ $17,6 \mu \mathrm{gPb} . \mathrm{m}^{-3}$ para as medianas dos meses de agosto e setembro, respectivamente. Assim, mesmo com uma redução de mais de $75 \%$, a concentração ainda é aproximadamente dez vezes maior que o valor limite tolerado pela EPA. Esse ponto corresponde à saída da chaminé, o que indica a necessidade de realizar ainda outras modificações no processo de purificação do ar emitido ao exterior.

\section{Conclusões}

Em trabalho anterior (Quiterio et al., 2001), verificou-se que a direção do vento, que as saídas de ar da RB e que as atividades desenvolvidas durante o período da coleta são fatores primordiais no planejamento e resultados das amostragens.

O uso de ar com indicador da concentração do chumbo no ar atmosférico nas circunvizinhanças da RB retrata o chumbo disperso na atmosfera, e não aquele acumulado, demonstrando uma contaminação ambiental recente.

A reformadora de baterias é a maior fonte de emissão estacionária de chumbo particulado para o ambiente na região. Neste trabalho, é mostrado que após um ano, a instalação do sistema de exaustão, as modificações no processo

\section{Figura 2}

Concentração de chumbo nas amostras de ar antes e após modificações na reformadora de baterias para cada ponto de amostragem selecionado.

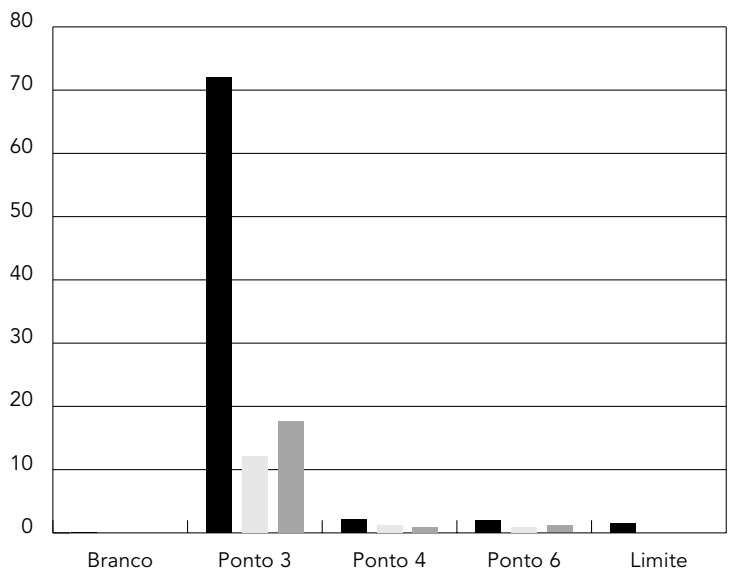

A primeira barra representa o valor médio antes das modificações, e a segunda e terceira barras representam os valores médios para os meses de agosto e setembro, respectivamente.

de produção e as melhorias das instalações promoveram uma significativa redução na emissão do metal para o ambiente. Contudo, os valores obtidos no Ponto 3, onde a concentração ainda está acima do valor limite tolerado pela EPA, indicam que ainda será necessário que se realizem outras modificações nas instalações e processo de produção e, posteriormente, um novo monitoramento do local.

Os resultados deste trabalho estão sendo utilizados para conscientizar os empresários quanto à importância da adoção de um SE eficiente e orientar a implantação de ações com o intuito de reduzir a poluição ambiental. Acredita-se que a continuidade deste trabalho contribuirá para que os responsáveis pelas empresas implantem mudanças que favoreçam a saúde dos trabalhadores e da população em geral.

\section{Agradecimentos}

Os autores agradecem ao Conselho Nacional de Desenvolvimento Científico e Tecnológico e ao Centro de Estudos da Saúde do Trabalhador e Ecologia Humana, Escola Nacional de Saúde Pública, Fundação Oswaldo Cruz, pelo apoio recebido para a realização deste trabalho. 


\section{Referências}

BAIRD, C., 1995. Environmental Chemistry. New York: W. H. Freeman.

CARB (California Air Resources Board), 1989. Information on Substances for Review as Toxic Air Contaminants. Report ARB/SSD/89-01. Sacramento: CARB.

CICT (Centro de Informação Científica e Tecnológica), 1999. Localização das Fábricas de Baterias do Município do Rio de Janeiro. Rio de Janeiro: CICT, Fundação Oswaldo Cruz.

EPA (Environmental Protection Agency), 1998. Guidance for Sitting Ambient Air Monitors Around Stationary Lead Sources USA. 27 May $1998<\mathrm{http}$ // www.epa.gov:80/ttnamtil/files/ambient/criteria/rel docs/ pbgde997.pdf>.

EPA (Environmental Protection Agency), 1999. Method 3015A, Microwave Assisted Acid Digestion of Aqueous Samples and Extracts. CD-ROM. Washington, DC: EPA.

GALVÃO, L. A. \& COREY, G., 1989. Plomo. Série Vigilancia. v. 8. México, DF: Organización Mundial de la Salud/Organización Panamericana de la Salud/Centro Americano de Ecología Humana y Salud.

LANDRIGAN, P. J.; GEHLBACH, S. H.; ROSENBLUM, B. F.; SHOULTS, J. M.; CANDELARIA, R. M.; BARTHEL, W. F.; LIDLLE, J. A.; SMRER, A. L. \& STAEHLING, N. W., 1975. Epidemic lead absorption near an ore smelter: The role of particulate lead. New England Journal of Medicine, 292:123129.

MARQUES, J., 1972. Apontamentos de Climatologia. Rio de Janeiro: Universidade Federal do Rio de Janeiro. (mimeo.)

MATTE, T. D.; FIGUEROA, J. P.; OSTROWSKI, S.; BURR, G.; JACKSON-HUNT, L.; KEENLYSIDE, R. A. \& BAKER, E. L., 1989. Lead poisoning among household members exposed to lead-acid battery repair shops in Kingston, Jamaica. International Journal of Epidemiology, 18:874-881.

MOREIRA, M. F. R., 1993. Determinação Direta de Cádmio em Sangue Total e Urina por Espectrometria de Absorção Atômica no Forno de Grafite. Dissertação de Mestrado, Rio de Janeiro: Departamento de Química, Pontifícia Universidade Católica do Rio de Janeiro.
NIST (National Institute of Standards and Technology), 1997. Certificate of Analysis. Standard Reference Material 3087a. Gaithersburg: NIST.

PINHEL, A., 2000. Condições Climáticas da Cidade do Rio de Janeiro no Ano de 1999 e 2000. Rio de Janeiro: Laboratório de Meteorologia, Universidade do Estado do Rio de Janeiro.

QUITERIO, S. L., 2000. Levantamento dos Níveis de Chumbo nas Circunvizinhanças de uma Fonte Estacionária de Emissão. Dissertação de Mestrado, Rio de Janeiro: Departamento de Físico-Química, Universidade Federal do Rio de Janeiro.

QUITERIO, S. L.; SILVA, C. R. S.; VAITSMAN, D. S.; MARTINHON, P. T.; MOREIRA, M. F. R.; ARAÚJO, U. C.; MATTOS, R. C. O. C. \& SANTOS, L. S. C., 2001. Uso da poeira e do ar como indicadores de contaminação ambiental em áreas circunvizinhas a uma fonte de emissão estacionária de chumbo. Cadernos de Saúde Pública, 17:501-508.

RTI (Research Triangle Institute), 1999. Toxicological Profile for Lead. Atlanta: Agency for Toxic Substances and Disease Registry, Public Health Services, U.S. Department of Health and Human Services.

SKERFVING, S., 1993. Inorganic lead. In: Criteria Documents from the Nordic Expert Group (B. B. Lundberg, ed.), pp. 125-238, Stockholm: International Labor Organization.

VAHTER, M. \& SLORACH, S., 1990. Exposure Monitoring of Lead and Cadmium: An International Pilot Study Within the WHO/UNEP. Stockholm: Human Exposure Assessment Location (HEAL) Program, Global Environment Monitoring System/World Health Organization.

YEH, C. Y.; CHIOV, H. Y.; CHEN, R. Y.; YEH, K. H.; JENG, W. L. \& HAN, B. C., 1996. Monitoring lead pollution near a storage battery recycling plant in Taiwan, Republic of China. Archives of Environment Contamination and Toxicology, 30:227-234.

Recebido em 21 de março de 2002

Versão final reapresentada em 27 de agosto de 2002 Aprovado em 22 de novembro de 2002 